\title{
Towards measurement of outcome for patients with varicose veins
}

\author{
Andrew M Garratt, Lesley M Macdonald, Danny A Ruta, Ian T Russell, \\ J Kenneth Buckingham, Zygmunt H Krukowski
}

\begin{abstract}
Objective-To develop a valid and reliable outcome measure for patients with varicose veins.

Design-Postal questionnaire survey of patients with varicose veins.

Setting-Surgical outpatient departments and training general practices in Grampian region.

Subjects-373 patients, 287 of whom had just been referred to hospital for their varicose veins and 86 who had just consulted a general practitioner for this condition and, for comparison, a random sample of 900 members of the general population.
\end{abstract}

Main measures-Content validity, internal consistency, and criterion validity.

Results-281(76\%) patients (mean age $45 \cdot 8 ; 76 \%$ female) and $542(60 \%)$ of the general population (mean age $47.9 ; 54 \%$ female) responded. The questionnaire had good internal consistency as measured by item-total correlations. Factor analysis identified four important health factors: pain and dysfunction, cosmetic appearance, extent of varicosity and complications. The validity of the questionnaire was demonstrated by a high correlation with the SF-36 health profile, which is a general measure of patients' health. The perceived health of patients with varicose veins, as measured by the SF-36, was significantly lower than that of the sample of the general population adjusted for age and a lower proportion of women.

Conclusion-A clinically derived questionnaire can provide a valid and reliable tool to assess the perceived health of patients with varicose veins.

Implications-The questionnaire may be used to justify surgical treatment of varicose veins.

(Quality in Health Care 1993;2:5-10)

\section{Introduction}

Varicose veins are common, with an estimated prevalence of about $8 \%$ in men and $16 \%$ in women. ${ }^{1}$ Their treatment imposes substantial costs on the NHS, whether measured by operations and bed days ${ }^{2}$ or financially. ${ }^{3} 4$

At a time when health care systems in the United Kingdom and abroad are trying to contain the rising costs of health care, clinicians are increasingly required to show that the care they deliver is effective and efficient. In the United States, for example, the state of Oregon has attempted to introduce a system of health care rationing that gives priority to those medical conditions for which treatment has proven benefit. ${ }^{5}$ In the United Kingdom the government has introduced managed competition into the NHS. ${ }^{6}$ In this internal market health authorities and fundholding practices are expected to purchase cost effective care from competing provider units.

To achieve this purchasers will need valid and reliable measures of patient outcome. There are few such measures for varicose veins, which may help to explain why there is no consensus about the relative merit of the two main forms of treatment - namely, injection sclerotherapy and surgery. ${ }^{7-11}$ Consequently, treatment for this condition has been given a low priority by some health authorities; North East Thames Regional Health Authority, for example, has advised its districts to curtail treatment for varicose veins when there is no "overriding clinical need." 12

Health profiles are general measures that assess health status across a range of separate dimensions like physical function, social function, mental health, and pain. The SF-36 health profile, ${ }^{13}$ for example, has been used widely in the United States and increasingly in the United Kingdom. ${ }^{14}$ The reliability and validity of this 36 item questionnaire in a patient population has been confirmed by recent studies in the United States ${ }^{15} 16$ and in a general practice population in the United Kingdom. ${ }^{14}$ The developers of the profile recommend using measures of health status specific to the condition in conjunction with general measures. A specific measure of health for varicose veins that asks specific questions about symptoms and signs and assesses the specific effect of varicose veins on health has potential advantages over more general measures.

(1) It may be more sensitive to small yet clinically significant changes in health status.

(2) It may have greater power to discriminate between patients whose health is only mildly affected by their varicose veins.

(3) It will contain questions that clinicians ask routinely when taking a clinical history (for example, "Does the appearance of your varicose veins cause you concern?" "How much ankle swelling have you had?") and will therefore be acceptable to them.

(4) The questions will be familiar to patients, 
and the resulting questionnaire will present them with few difficulties.

The development of a clinically based specific outcome measure for patients with varicose veins therefore has much to contribute to the debate about resource allocation within the NHS. This paper explores the extent to which such a measure reflects patients' perceptions of their health. We describe the development of the questionnaire and compare the responses with those to the SF-36 health profile. The full effect of varicose veins on patient wellbeing was assessed by comparing the health status of a sample of patients with varicose veins with that of a random sample of the general population.

\section{Methods}

DEVEI OPING' THE QUESTIONNAIRE

An outcome measure should be both valid and reliable. Validity is the extent to which a questionnaire measures what is intended. ${ }^{17}$ Reliability is the extent to which measurements on the same individual are similar under different circumstances. ${ }^{17}$ In this paper, rather than measure the same individual under different circumstances, we assessed reliability through internal consistency - that is, the extent to which similar questions give consistent responses. ${ }^{17}$

In developing the questionnaire we followed five steps recommended by Streiner and Norman. ${ }^{17}$

\section{Devising and scoring the questions}

The questionnaire (see appendix) was based on questions commonly used in the clinical assessment of patients with varicose veins, as indicated by a review of the clinical literature. The content validity of the questionnaire, which assesses whether the selected questions are representative of the domain of all relevant questions, ${ }^{17}$ was confirmed by two consultant surgeons. The two surgeons then scored the questionnaire independently; each allocated a score of zero to all response categories considered normal and scores to all other categories in proportion to their perceived contribution to severity.

The two sets of scores were then averaged and rescaled to yield total scores between zero and 100; zero is reserved for patients with no evidence of varicose veins and 100 for patients ticking the most severe response to each question (for example, "In the last two weeks my varicose veins caused me pain or ache for more than 10 days; severe ankle swelling; and to wear support stockings every day"). If a question is omitted by a patient the total score is calculated after removing the score for that question from the denominator. This ensures that patients can still score between zero and 100 if they omit questions that they consider inappropriate.

\section{Pretesting the questions}

To check for comprehensibility and lack of ambiguity we sent the resulting questionnaire to a small sample of patients with varicose veins, who were subsequently interviewed.

\section{Testing the questions}

Between March and June 1991 patients with varicose veins were identified in two ways: from all referral letters to the surgical outpatient departments in Grampian ${ }^{18}$ and by general practitioners from four large training practices in Grampian, whose patients were included only if the general practitioner did not refer them to a specialist during the recruitment period of the study. A questionnaire including the clinical questions, an anglicised version of the SF-36 health profile (A M Coulter, personal communication), and relevant sociodemographic questions was sent to the patients in general practice within two weeks of their initial consultation and to the referred patients before their first outpatient appointment. All patients not wishing to take part in the study were asked to return their questionnaires blank. Reminders were sent to non-responders after two weeks and again after four weeks.

Questions were considered for rejection if more than $80 \%$ of patients gave the same response because such questions are not sensitive enough to discriminate between different degrees of severity. ${ }^{17}$

\section{Assessing internal consistency}

We used two measures of internal consistency: Cronbach's alpha $^{19}$ (which measures the correlation between items - that is, questions) and item-total correlations (which compare scores for individual questions with the total weighted score). Questions yielding item-total correlations below 0.2 were considered for rejection..$^{20}$

However, questions that measure different aspects of health cannot be expected to correlate well with the total score. Factor analysis $^{21}$ was therefore used to identify separate health "factors" within the questionnaire. A factor was considered important if its "eigenvalue" (a statistical measure of its power to explain variation between patients) exceeded $1 \cdot 1^{22}$; and if it had face validity - that is, if it seemed "at face value" to be measuring a clinically recognisable aspect of patients' health. Questions that did not contribute significantly to any of these important factors were considered for rejection.

5 Finalising the questionnaire for clinical use Questions were included in the definitive questionnaire if they satisfied at least two of the following three criteria: all response categories ticked by less than $80 \%$ of patients; a significant coefficient within one of the important factors; and an item-total or relevant item-factor correlation above $0 \cdot 2$. Finally we checked that Cronbach's alpha for the resulting questionnaire exceeded 0.5 - the criterion that needs to be satisfied when comparing groups of patients. ${ }^{23}$

VALIDATING THE QUESTIONNAIRE

Criterion validity is the extent to which a new measure is correlated with established measures of the concept under study. ${ }^{17}$ We assessed the criterion validity of the definitive 
Table 1 Correlation between severity score for varicose veins and eight scales of SF-36 health profile ${ }^{13}$

\begin{tabular}{|c|c|}
\hline SF-36 dimensions and scales & Correlation with varicose vein score ${ }^{*}$ \\
\hline \multicolumn{2}{|l|}{ Functional status: } \\
\hline Physical functioning & -0.49 \\
\hline Social functioning & -0.44 \\
\hline Role limitations attributed to physical problems & $-0 \cdot 35$ \\
\hline Role limitations attributed to emotional problems & $-0 \cdot 41$ \\
\hline \multicolumn{2}{|l|}{ Wellbeing: } \\
\hline Mental health & $-0 \cdot 31$ \\
\hline Energy and fatigue & $-0 \cdot 38$ \\
\hline Pain & $-0 \cdot 44$ \\
\hline \multicolumn{2}{|l|}{ Overall evaluation of health: } \\
\hline General health perception & $-0 \cdot 25$ \\
\hline
\end{tabular}

questionnaire as a measure of health by comparing it with the SF-36 health profile, ${ }^{13}$ which measures health across three dimensions and includes eight separate scales (table 1). The responses to the questions on each scale are summed and rescaled to yield scores between zero and 100; zero is reserved for patients ticking the most severe response to each question and 100 for patients ticking the best possible response to each question (that is, the opposite of the severity score for varicose veins)

Patients' scores on the definitive questionnaire were compared with their scores on each of the eight scales within the SF-36 health profile. Stepwise multiple regression ${ }^{24}$ was then used to investigate the relation between the varicose veins score and all the SF-36 scales simultaneously. This analysis also included six sociodemographic variables: age, sex, marital status, age on completing education, home ownership, and employment status and an additional SF-36 variable measuring change in health over the past year.

COMPARISON WITH THE GENERAL POPULATION A random sample of 900 members of the general population selected from the electoral registers for Aberdeen served as a comparison group. They were sent a similar questionnaire, including the SF-36 health profile and sociodemographic questions but without the clinical questions relating to varicose veins. Their responses were adjusted to yield the same age-sex distribution as the varicose vein sample before comparison by weighted $t$ tests.

\section{Results}

DEVELOPING THE QUESTIONNAIRE

A pilot survey of 12 patients confirmed that the questionnaire was easy to complete, and follow up interviews confirmed that the questions were well understood. Although some patients had difficulty in completing the diagram in question 1 (see appendix), most were able to complete it without apparent difficulty. We therefore decided to include this question unaltered in the main survey and to determine empirically whether it should be discarded from the definitive questionnaire. Otherwise, only minor changes in wording were made.

In the main survey 373 patients were identified, 287 from referral letters and 86 by their general practitioners. In all, 34 patients returned blank questionnaires and 53 failed to respond; five questionnaires could not be delivered by the postal system. Thus 281 patients responded, yielding a response rate of $76 \%$. Their mean age was 45.8 years (range $19-83$ years); $212(76 \%)$ were female (the same proportion as in the original sample) and $215(76 \%)$ had just been referred to a surgical outpatient clinic.

The response frequencies in the main survey (table 2) show that questions 4 ("On how many days did you take pain killing tablets for your varicose veins?"), 7 ("Do you take 'water tablets' for ankle swelling?"), 10 ("Do you have a rash or eczema in the area of your ankle?"), and 11 ("Do you have a skin ulcer associated with your varicose veins?") were answered negatively by more than $80 \%$ of respondents and therefore provided little discrimination. Only question 3 ("At what time of the day were your varicose veins usually most painful or aching?") yielded an item-total correlation of less than $0 \cdot 2$ (table 2 ).

Of the final six columns of table 2 , the first four represent important health factors. A consultant surgeon ( $\mathrm{ZHK}$ ) confirmed that each corresponds to a clinically recognisable aspect of health - namely, pain and dysfunction (F1), cosmetic appearance (F2), extent of varicosity (F3), and complications

Table 2 Selection of questions for outcome questionnaire

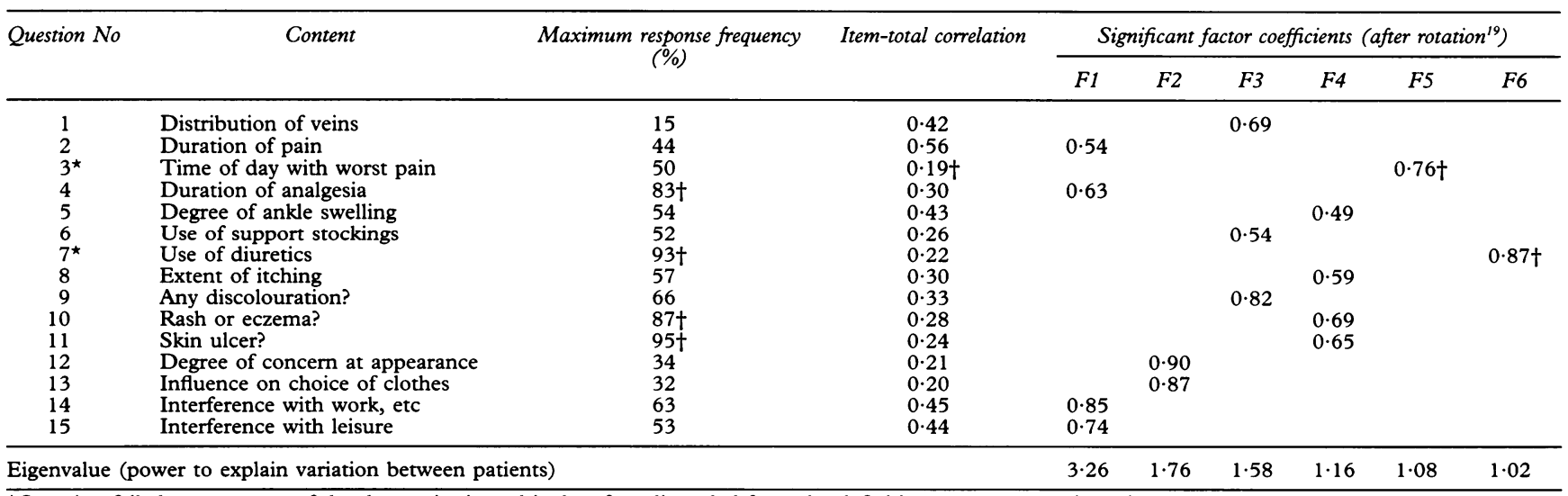

ॠQuestion failed to meet two of the three criteria and is therefore discarded from the definitive outcome questionnaire.

tQuestion failed to meet relevant criterion for inclusion in outcome questionnaire. 

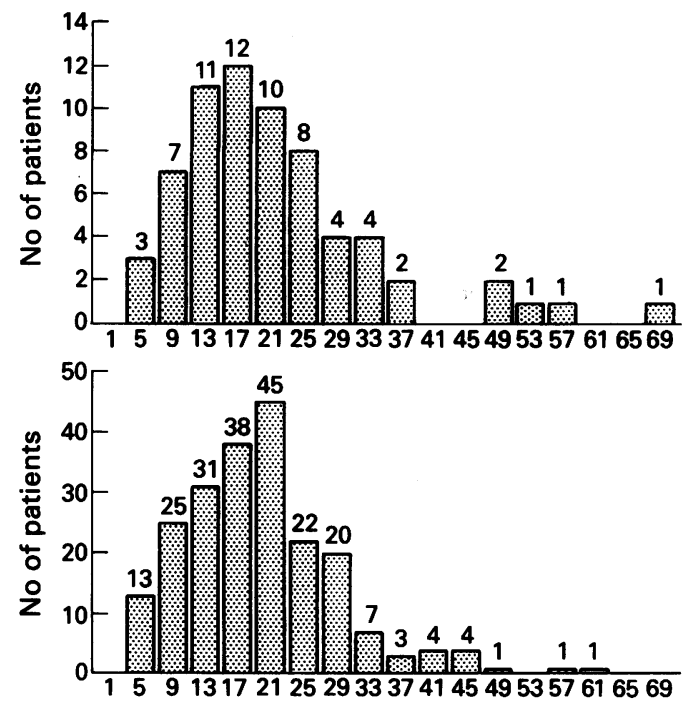

Severity scores for varicose veins (midpoint)

Fig 1 Distribution of severity scores for varicose veins. Top: non-referred patients $(n=66)$; bottom: referred patients $(n=215)$

(F4). In contrast, questions 3 ("At what time of the day were your varicose veins usually most painful or aching?") and 7 ("Do you take 'water tablets' for ankle swelling?") are isolated in factors $\mathrm{F} 5$ and F6 respectively. These two factors are unimportant (in the sense that their eigenvalues are less than $1 \cdot 1$ ) and each contains only one question; hence there is little evidence that they represent any clinically distinct health factor. Thus questions 3 and 7 failed two of the three criteria and were removed from the definitive questionnaire. This achieved a Cronbach's alpha statistic of 0.72 , thus satisfying the criterion proposed by Helmstadter. ${ }^{23}$

Figure 1 shows the distribution of the resulting varicose vein severity scores. For both referred and non-referred patients the scores are skewed but quite close to normality. For referred patients they ranged from 3.0 to 62.8 (median 19.1); for non-referred they ranged from 3.4 to 71.0 (median 19.3 ). That there is no significant difference between these two distributions may reflect variations in referral behaviour between general practitioners.

VALIDATING THE QUESTIONNAIRE

Table 1 shows that the varicose vein severity scores achieve highly significant negative correlations with all eight scales of the SF-36 health profile. Four of these correlations exceed 0.4 in absolute magnitude: those with physical functioning, pain, social functioning, and role limitations attributed to emotional problems; only the correlation with general

Table 3 Stepwise multiple regression of severity score for varicose veins on eight scales of SF-36 health profile ${ }^{13}$

\begin{tabular}{lcccc}
\hline \multicolumn{1}{c}{ SF-36 scale } & Regression coefficient & Standard error & $t$ & Significance \\
\hline Physical function $^{\star}$ & -0.115 & 0.032 & -3.53 & 0.0005 \\
Emotional limitations $^{\star}$ & -0.065 & 0.015 & -4.19 & 0.0000 \\
Pain $^{\star}$ & -0.092 & 0.029 & -3.17 & 0.0017 \\
(Constant) & 39.7 & 2.2 & 18.04 & 0.0000 \\
\hline
\end{tabular}

ॠThese variables account for $32 \%$ of the variation in the varicose vein scores $\left(R^{2}=0 \cdot 317\right)$. health perceptions falls below 0.3 in magnitude. These correlations suggest that the questionnaire is picking up adverse effects of varicose veins on all eight of the health scales that make up the SF-36 health profile.

However, these eight scales are also known to be correlated with each other. ${ }^{25}$ To summarise the relation between the varicose vein severity score and the SF-36 health profile while taking account of these correlations we used stepwise multiple regression. ${ }^{24}$ Table 3 shows that three of the SF-36 scales - physical functioning, role limitations attributed to emotional problems, and pain - were sufficient to explain $32 \%$ of the variation in the varicose vein scores. Once these three scales were included in the regression equation, no other scale or sociodemographic variable could improve the fit of the regression. This suggests that the questionnaire explains a substantial proportion of the non-random variation in patients' perceived health.

COMPARISON WITH THE GENERAL POPULATION Finally, SF-36 health profile scores for the patients with varicose veins were compared with those of a random sample of the general population (fig 2). Of the 900 subjects sampled, 542(60\%) returned the questionnaire. Their ages ranged from 18 to 91 years (mean 47.9 ) years; $359(54 \%$ ) were female. The responses from this general sample were therefore weighted to adjust for their higher average age (by two years) and their lower proportion of women (by $21 \%$ ). The patients with varicose veins reported significantly worse health on five of the eight SF-36 scales: role limitations attributed to physical problems and those attributed to emotional problems, energy and fatigue, pain, and general health. There were no significant differences on the three remaining scales: physical functioning, social functioning, and mental health.

\section{Discussion}

We have shown that it is possible to take a comprehensive set of clinical questions for varicose veins and, by subjecting them to a rigorous process of development, to generate an outcome questionnaire that possesses good internal consistency. Furthermore the validity of this questionnaire as a measure of the effect of varicose veins on health has been confirmed by its high negative correlation with all the varied aspects of perceived health measured by the SF-36 health profile. The three SF-36 scales which best summarised the relation between the varicose vein score and patients' perceptions of their health were those measuring physical functioning, role limitations due to emotional problems, and pain. These are aspects of health which might be expected to be affected by varicose veins.

That only two clinical questions failed to meet our stringent criteria for inclusion in the definitive questionnaire reflects well on the validity of clinical judgement in the assessment of patients' health and wellbeing. Clinically derived outcome measures have been criticised for concentrating on symptoms and 


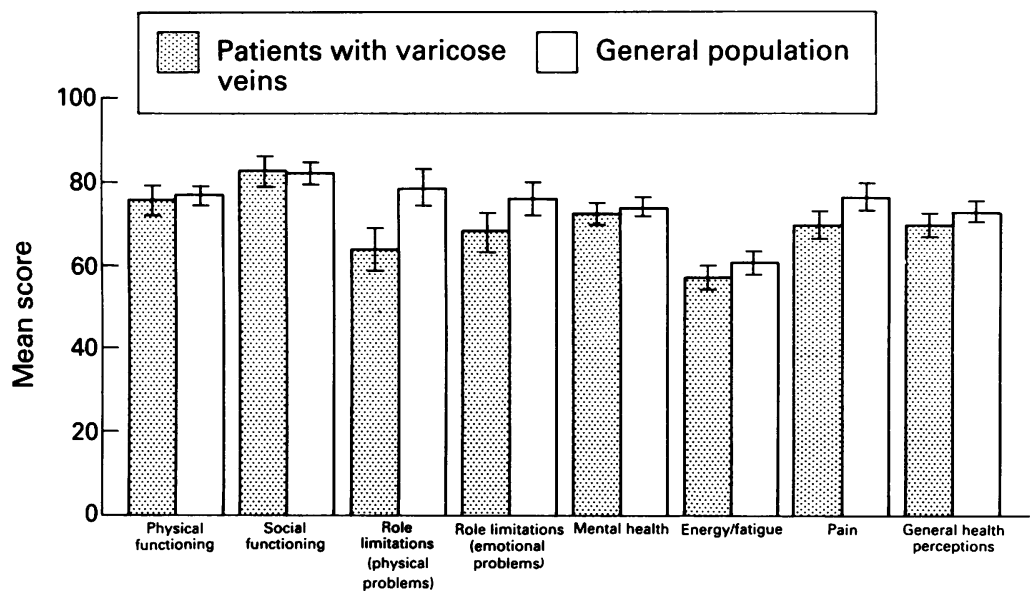

Differences between groups for role limitations (physical and emotional problems) and pain significan at $1 \%$ and for energy/fatigue and general health perceptions at $5 \%$ (weighted $t$ test)

Fig 2 Mean scores of eight scales of SF-36 health profile in patients with varicose veins $(n=281)$ and random sample of general population $(n=542)$ adjusted for age and sex. Bars are $95 \%$ confidence intervals justify their claim on scarce NHS resources by demonstrating health gains for their patients.

We thank the staff of Inverurie, Portlethen, Rubislaw Place, and Westhill practices for their help, and Mr R A Keenan for scoring the outcome questionnaire. This research and the Health Services Research Unit are both funded by the Chief Scientist Office at the Scottish Office Home and Health Department; however, the opinions expressed are those of the authors and not the SOHHD.

1 Chant ADB, Jones HO, Weddell JM. A comparison of surgery and injection-compression sclerotherapy. Lancet 1972;ii:1188-91.

2 Information and Statistics Division. Scottish health statistics 1991. Edinburgh: Common Services Agency for the Scottish Health Service, 1991.

3 Piachaud D, Weddell JM. The economics of treating varicose veins. Int f Epidemiol 1972;1:287-94.

4 Beresford SAA. Varicose veins: a comparison of surgery and injection-compression sclerotherapy - five year and injection-compression scler

5 Dixon J, Welch HG. Priority setting: lessons from Oregon Lancet 1991;337:891-4.

6 Secretaries of State for Health, Wales, Northern Ireland, and Scotland. Working for patients. London: HMSO, 1989. (Cmnd 555.)

7 Nabatoff RA, Stark DCC. The treatment of varicose veins. Lancet 1973; i:201-2.

signs, thus describing only limited aspects of patients' health. ${ }^{26}$ If the SF-36 health profile genuinely reflects patients' perceptions of health, however, our results show a high level of agreement between the perceptions of doctors and patients.

Varicose veins are often considered a relatively unimportant condition, to the extent that one health authority has considered curtailing treatment. ${ }^{12}$ This study showed that there are significant differences in health between patients with varicose veins and the general population. By assessing these patients after treatment, we plan to use the SF-36 health profile and our clinical questionnaire in combination to estimate the extent of any improvement in health. Because the SF-36 health profile is a general measure the information it provides could be used to throw light on the cost effective allocation of resources between treatments and across conditions. Because of its restricted focus, however, our clinically based questionnaire may be more sensitive in showing change over time and in discriminating between patients with mild symptoms. Thus it may be more useful in managing individual patients. We shall address these issues by following up our sample of patients at six months and one year.

In conclusion, a clinically based questionnaire such as we have described, together with a general measure of health such as the SF-36 health profile, can provide a valid and reliable tool to assess the perceived health of patients presenting with varicose veins. This may yet enable surgeons who treat varicose veins to
8 Doran FSA, White M. A clinical trial designed to discover if the primary treatment of varicose veins should be by Fegan's method or by operation. Br $\mathcal{f}$ Surg 1975;62: $72-7$

9 Hobbs JT. Surgery and compression sclerotherapy in the treatment of varicose veins. Arch Surg 1974;109:793-7.

10 Jakobsen BH. The value of different forms of treatment for varicose veins. Br f Surg 1979;66:182-4.

11 Munn SR, Morton JB, MacBeth WAAG, McLeish AR. To strip or not to strip the long saphenous vein? A varicose veins trial. Br $\mathcal{F}$ Surg 1979;66:182-4.

12 Dean $M$. London perspective: end of a comprehensive NHS? Lancet 1991;337:351-2.

13 Ware JE, Sherbourne CD. The MOS 36-item short-form health survey (SF-36). I. Conceptual framework and item selection. Med Care 1992;30:473-83.

14 Brazier JE, Harper R, Jones NMB, O'Cathain A, Thomas KJ, Usherwood, et al. Validating the SF-36 Thomas KJ, Usherwood, et al. Validating the SF-36 health survey questionnaire: new ou
primary care. $B M \mathcal{F}^{1992 ; 305: 160-4}$.

15 McHorney CA, Ware JE, Raczek AE. The MOS 36-item short-form health survey (SF-36). II. Psychometric and clinical tests of validity in measuring physical and menta health constructs. Med Care (in press).

16 McHorney CA, Ware JE, Rogers W, Raczek AE, Racel JF. The validity and relative precision of MOS short and long-form health status scales and Dartmouth COOP charts. Medical Care Suppl 1992;30:MS253-65.

17 Streiner GL, Norman DR. Health measurement scales: a practical guide to their development and use. Oxford: Oxford practical guide to their deve.

18 Grimshaw JM, Russell IT, Taylor RJ. Grampian Referral Initiatives Project: data collection and the interpretation and Initiatives Project: data collection and the interpretation and
feedback of referral rates. Aberdeen: Health Services feedback of referral rates. Aberdeen: Health Services
Research Unit, University of Aberdeen, 1992. Research Unit, Univers
(Occasional paper 13.)

19 Cronbach LJ. Coefficient alpha and the internal structure of tests. Psychometrika 1951;16:297-334. 1986. Springer-Verlag, 1986.

22 Jolliffe IT, Morgan BJT. Principal component analysis and exploratory factor analysis. Statistical Methods in Medical Research 1992;1:69-95.

23 Helmstadter GC. Principles of psychological measurement. New York: Appleton-Century-Crofts, 1964.

24 Armitage P, Berry G. Statistical methods in medical research. 2nd ed. Oxford: Blackwell, 1987.

25 Stewart AL, Hays RD, Ware JE. The MOS short-form general health survey. Med Care 1988;26:724-33. of hypotensive drugs on the quality of life. $\mathcal{f} R$ Coll Gen Pract 1982;32:103-5.
20 Kline P. $A$ handbook of test construction. London: Methuen,

21 Jolliffe IT. Principal component analysis. New York:

26 Jachuck SJ, Brierley H, Jachuck S, Willcox PM. The effect 


\section{Appendix \\ Clinical varicose veins questionnaire}

1 Please draw in your varicose veins in the diagram(s) below:

2 In the past two weeks, for how many days did your varicose veins cause you pain or ache? (Please tick one box for each leg)

3 In the past two weeks at what time of day were your varicose veins usually most painful or aching?

(Please tick one box)

Not painful at all

No particular time

In the afternoon and/or evening At night

4 During the past two weeks, on how many days did you take painkilling tablets for your varicose veins?

(Please tick one box)

5 In the past two weeks, how much ankle swelling have you had? (Please tick one box)

Moderate ankle swelling (for example causing you to sit with your feet up whenever possible) Severe ankle swelling (for example causing you difficulty putting on your shoes)

6 In the past two weeks, have you worn support stockings or tights?

(Please tick one box for each leg)

Yes, those I bought myself without a doctor's prescription Yes, those my doctor prescribed for me, which I wear occasionally Yes, those my doctor prescribed for me, which I wear every day

7 Do you take "water tablets" for ankle swelling?

(Please tick one box)

8 In the past two weeks, have you had any itching in association with your varicose veins? (Please tick one box for each leg)

Yes, but only above the knee Yes, but only below the knee Yes, both above and below the knee

9 Do you have purple discoloration caused by areas of tiny blood vessels in the skin, in association with your varicose veins?

(Please tick one box for each leg)

10 Do you have a rash or eczema in the area of your ankle? (Please tick one box for each leg)

11 Do you have a skin ulcer associated with your varicose veins?

12 Does the appearance of your varicose veins cause you concern? (Please tick one box)

13 Does the appearance of your varicose veins influence your choice of clothing, including tights? (Please tick one box)

Occasionally Often Always

14 During the past two weeks have your varicose veins interfered with your work or housework or other daily activities?

(Please tick one box)

I have been able to work but my work has suffered to a slight extent I have been able to work but my work has suffered to a moderate extent My veins have prevented me from working one day or more

15 During the past two weeks have your varicose veins interfered with your leisure activities (including sport, hobbies and social life)?

(Please tick one box) 